$90 \%$ of ACPA-lgGs harbour $\mathrm{N}$-linked glycans in the antibody variable (V) domain The corresponding $\mathrm{N}$-glycosylation sites in the amino acid backbone of ACPA Vregions result from somatic hypermutation, a T cell-dependent process. Notably, both genetic evidence and data obtained from the analysis of serum ACPA indicate that T-cells drive the maturation of the ACPA-response prior to the onset of arthritis.

Objectives: We investigated whether ACPA-IgG carry V-domain N-glycans prior to the development of arthritis and whether the occurrence of such glycans predicts the transition from pre-disease autoimmunity to overt RA.

Methods: Two independent sets of serum samples were obtained from RA patients and from ACPA-positive first-degree relatives (FDR) of RA-patients $(n=126)$ of an Indigenous North American (INA) population with high incidence rates of ACPA-positive RA. These samples comprised cross-sectional and longitudinal samples of individuals who did or did not transition to inflammatory arthritis. Serum ACPA-IgG were affinity-purified and subjected to enzymatic glycan release and UHPLC-based glycan analysis.

Results: ACPA-IgG V-domain glycosylation could be detected in RA patients and in FDR of RA patients. In both datasets, FDR-derived ACPA-lgG displayed markedly lower levels of V-domain glycans $(<50 \%)$ compared to ACPA-IgG from RA-patients. Notably, FDRs that later developed RA showed extensive V-domain glycosylation before the onset of arthritis. Moreover, the degree of ACPA-lgG Vdomain glycosylation in FDRs was strongly associated with future development of RA (HR: 6.07 [95\% Cl: 1.46-25.2]; $p=0.013$ ).

Conclusion: Glycosylation of the ACPA-IgG V-domain can be detected prior to the onset of disease. Extensive glycosylation is present in a subset of predisposed FDRs of INA RA patients. The presence of this feature substantially increases the risk of RA development. These observations fit well with a pivotal role for $T$ cells in the selection and expansion of ACPA-expressing B cells, possibly by facilitating the introduction of $\mathrm{N}$-glycosylation sites in ACPA-lgG V-domains. Moreover, glycosylation of the ACPA-IgG V-domain represents a predictive marker for RA development in ACPA-positive individuals and may serve to better time and target preventive therapeutic interventions.

Disclosure of Interests: Lise Hafkenscheid: None declared, Emma C. de Moel: None declared, Irene Smolik: None declared, Xiaobo Meng: None declared, Stacy Tanner: None declared, Bas C. Jansen: None declared, Albert Bondt: None declared, Manfred Wuhrer: None declared, Thomas Huizinga Consultant for: Merck, UCB, Bristol Myers Squibb, Biotest AG, Pfizer, GSK, Novartis, Roche, Sanofi-Aventis, Abbott, Crescendo Bioscience Inc., Nycomed, Boeringher, Takeda, Zydus, Epirus, Eli Lilly, Rene Toes Grant/research support from: Sanofi, Hani El-Gabalawy: None declared, Hans Ulrich Scherer Grant/research support from: Sanofi, BMS

DOI: 10.1136/annrheumdis-2019-eular.7067

\section{OP0296 1 COMPARISON OF TRANSCRIPTOMIC PROFILES BETWEEN PAIRED JOINT BIOPSIES FROM RHEUMATOID ARTHRITIS PATIENTS}

Clément Triaille $^{1,2}$, Louise Vansteenkiste ${ }^{1}$, Laurent Meric de Bellefon ${ }^{1,3}$, Adrien Nzeusseu ${ }^{1,3}$, Christine Galant ${ }^{1,4}$, Patrick Durez ${ }^{1,3}$, Bernard Lauwerys s $^{1,3}$ ${ }^{1}$ Pôle de pathologies rhumatismales systémiques et inflammatoires, Institut de Recherches Expérimentales Cliniques, Université catholique de Louvain, Brussels, Belgium; ' 2 Service d'Hématologie et Oncologie pédiatrique, Cliniques Universitaires Saint-Luc, Brussels, Belgium; ${ }^{3}$ Service de Rhumatologie, Cliniques Universitaires Saint-Luc, Brussels, Belgium; ${ }^{4}$ Service d'Anatomie Pathologique, Cliniques Universitaires Saint-Luc, Brussels, Belgium

Background: Rheumatoid Arthritis (RA) is a chronic and heterogenous condition characterized by inflammatory involvement of the synovial membrane in multiple joints. Synovial biopsies are used in research setting in order to identify diagnostic and theranostic markers. Many studies have shown a high degree of heterogeneity in histological and transcriptomic profiles between patients.

Objectives: We wanted to explore histological and transcriptomic profile of synovial biopsies across pairs of joints in the same patients to assess heterogeneity at the individual level.

Methods: Synovial biopsies were performed simultaneously in one small and one large joint per patient using needle-arthroscopy for the knee and US-guided needle biopsy for the hand or foot. Synovium from individuals with osteoarthritis (OA) were used as control. Paraffine-embedded samples were stained for CD3, CD 20, CD 68 and CD138. Whole-tissue RNA was extracted and hybridized on GeneChip Human Genome U133 Plus 2.0 Array (Affymetrix). The samples were tested for RNA integrity by Bioanalyzer (Agilent). Normalization and statistical analyzes were performed on Genespring. Pathway analysis were performed using DAVID.

Results: 10 RA patients were included (females: 10/10, ACPA/RF positivity: 8/ 10 , mean age $( \pm$ SEM): $54.4( \pm 4.4$ ) years, mean disease duration $( \pm S E M): 13.3$ $( \pm 3.7)$ years, mean DAS28CRP $( \pm$ SEM): $5.01( \pm 0,34)$, mean HAQ $( \pm$ SEM $): 1.7$ $( \pm 0.28))$.
Quantification of histological markers did not show differences in population of macrophages, plasmocytes, $T$ and B Cells, across pairs of joints.

After correction for multiple comparisons, no transcripts were differentially expressed between large and small joints. Similarly, we did not find any significan difference in the expression of transcripts involved in pathways (TCR-activation and cell-division) specifically overexpressed in RA compared to OA synovial tissue.

In order to increase our ability to observe pair-wise differences in gene expression profiles, we studied correlations between transcripts significantly overexpressed in RA compared to OA joints with a fold change $>2(n=581)$ and clinical or biological markers of disease activity (DAS28-CRP, CRP, Physician Global Assessment of disease activity). Similar patterns of correlations indicated that disease activity was not driven by different pathways in small versus large joints.

Conclusion: This study is an important methodological milestone in the field of synovial biopsies, as it indicates that cellular and molecular alterations occurring in RA synovitis are similar across small and large joints from the same patient. Hence, biopsy of a single joint is representative and can be used to explore pathogenic processes or potential biomarkers in RA.

Acknowledgement: This work was funded in part by grants from Cap48 (RTBF), Fonds National de la Recherche Scientifique (FNRS, Communauté française de Belgique) and Fondation Saint-Luc.

Disclosure of Interests: Clément Triaille: None declared, Louise Vansteenkiste: None declared, Laurent Meric de Bellefon: None declared, Adrien Nzeusseu: None declared, Christine Galant: None declared, Patrick Durez Speakers bureau: Bristol-Myers Squibb, Eli Lilly, Sanofi, Celltrion, Bernard Lauwerys: None declared

DOI: 10.1136/annrheumdis-2019-eular.7405

\section{OP0297} ABERRANT ADENOSINE TO INOSINE RNA EDITING IN ACTIVE RHEUMATOID ARTHRITIS

Nikolaos Vlachogiannis $^{1,2}$, Aikaterini Gatsiou², Kimon Stamatelopoulos ${ }^{3}$, Maria Tektonidou ${ }^{1}$, Petros Sfikakis ${ }^{1}$, Konstantinos Stellos ${ }^{2} .{ }^{1}$ National and Kapodistrian University of Athens Medical School, First Department of Propaedeutic Internal Medicine and Joint Rheumatology Program, Athens, Greece; ${ }^{2}$ Newcastle University, Faculty of Medical Sciences, Institute of Genetic Medicine, Newcastle upon Tyne, United Kingdom; ${ }^{3}$ National and Kapodistrian University of Athens Medical School, Department of Clinical Therapeutics, Athens, Greece

Background: Adenosine to inosine (A-to-I) RNA editing is a widespread posttranscriptional RNA modification mainly located in repetitive Alu elements and mediated by the enzyme adenosine deaminase acting on RNA-1 (ADAR1). A-toRNA editing controls various aspects of RNA metabolism, which may affect tissue-specific gene expression. Although deregulation of RNA editing has been previously reported in various human diseases including cardiovascular disease and cancer (Stellos et al., Nat Med, 2017; Liu et al., Nat Med, 2019 and Ishizuka et al., Nature, 2019), its role in autoimmune diseases and especially in rheumatoid arthritis $(R A)$ remains unknown.

Objectives: To study whether A-to-I RNA editing is involved in the pathogenesis of RA and to determine the impact of anti-rheumatic treatment on RNA editing Methods: We first analysed the expression of ADAR1 in 185 RA synovial tissues versus 76 healthy/osteoarthritic synovia derived from 4 independent RNAsequencing and microarray datasets. We validated the findings in periphera blood mononuclear cells (PBMCs) derived from 19 patients with active RA vs 14 controls and performed an additional ADAR1-isoform analysis (ADAR1p110/ ADAR1p150) by RT-qPCR. Further, we studied in single nucleotide level the A-toI RNA editing levels of the pro-inflammatory gene cathepsin S (CTSS) 3'-untrans lated region (3'UTR), a matrix degradation enzyme which is a well-established target of ADAR1, by Alu Sanger sequencing and RNA editing analysis. Last, we examined the effect of anti-rheumatic treatment on RNA editing

Results: Expression of the RNA editor ADAR1 was significantly increased in RA synovium compared to healthy or osteoarthritic synovium. Similarly, a significant increase of ADAR1, mainly due to an increase of the interferon-inducible ADAR1p150 isoform, was observed in PBMCs from active RA. Next, we studied the RNA editing levels in PBMCs from active RA patients before and after 12 week treatment versus controls. RNA editing of CTSS 3'UTR AluSx ${ }^{+}$was increased in active RA (6-47\% increase in editing rate of 8 individual adenosines, all $\mathrm{P}<0.05$ ). Increased CTSS mRNA expression in RA PBMCs was associated with both ADAR1p150 expression $(r=0.623, P=0.004)$ and RNA editing rate of 12 individual adenosines ( $r$ range $0.45-0.72, P<0.05$ for all) located within the CTSS 3 ' UTR AluS $x^{+}$. The correlation between CTSS and ADAR1 was also observed in synovial tissue. Notably, ADAR1p150 expression and RNA editing rate reached control levels after 12-week treatment with methotrex ate \pm corticosteroids and/or biologics in patients with good clinical response (EULAR responders) but remained unchanged in the EULAR moderate/nonresponders. 
Conclusion: ADAR1-mediated RNA editing is increased in active RA inducing the expression of pro-inflammatory genes thus representing a novel drug response biomarker and a potential therapeutic target in EULAR moderate/nonresponders.

\section{REFERENCES:}

[1] Stellos, et al., Nat Med 2016;22:1140-50. doi:10.1038/nm.4172

[2] Liu, et al., Nat Med 2019;25:95-102. doi:10.1038/s41591-018-0302-5

[3] Ishizuka, et al., Nature 2019;565:43-8. doi:10.1038/s41586-018-0768-9

Disclosure of Interests: None declared

DOI: 10.1136/annrheumdis-2019-eular.4710

\section{FRIDAY, 14 JUNE 2019}

\section{Novel biomarkers in RMDs - next steps towards clinical implementation}

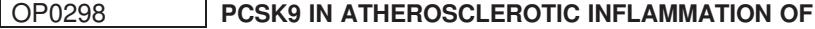 LUPUS PATIENTS AND MURINE MODEL OF LUPUS WITH ATHEROSCLEROSIS}

Chenglong Fang ${ }^{1}$, Tingting Luo ${ }^{2} .{ }^{1}$ Second Affiliated Hospital of Fujian Medical University, Department of Rheumatology, Quanzhou, China; ${ }^{2}$ Second Affiliated Hospital of Fujian Medical University, Quanzhou, China

Background: Systemic lupus erythematosus (SLE) patients have tendencies of accelerated atherosclerosis (AS), which is refractory to statins. Proprotein convertase subtilisin/kexin type 9 (PCSK9) is a new therapeutic target for AS for its dual mechanisms in lipids metabolism and inflammation. PCSK9 inhibitors had proved to be highly promising cardiovascular disease (CVD) drugs [1]. Our previous study suggested that Toll-like recetor 4(TLR4) signal participates in the atherosclerotic inflammation of murine model of lupus with AS [2].

Objectives: To investigate the role of PCSK9 in atherosclerotic process of lupus and the association between TLR4 and PCSK9 in athergenic inflammation of murine model of lupus with AS.

\begin{tabular}{llll} 
Table 1 Characteristics of SLE patients and controls. & \\
\hline Variables & SLE & Healthy controls & p-value \\
\hline Number & 90 & 50 & NA \\
Female/Male & $82 / 8$ & $43 / 7$ & $>0.05$ \\
Age(years) & $33(29-42)$ & $32(28-44)$ & $>0.05$ \\
Disease duration(years) & $6(3-10)$ & NA & NA \\
SLEDAl & $6(2-8)$ & NA & NA \\
Cholesterol(mmol/l) & $4.52(3.85-5.37)$ & $4.52(3.93-5.60)$ & $>0.05$ \\
LDL cholesterol(mmol/I) & $2.47(1.84-3.16)$ & $2.58(2.03-3.06)$ & $>0.05$ \\
Triglycerides(mmol/l) & $1.16(0.79-1.61)$ & $1.34(0.87-1.56)$ & $>0.05$ \\
HDL cholesterol(mmol/l) & $1.47(1.23-1.73)$ & $1.61(1.36-1.91)$ & $>0.05$ \\
CIMT thickening & $27 / 90(30.00 \%)$ & $7 / 50(14.00 \%)$ & 0.03 \\
Treatment & & & \\
HCQ use & $90 / 90(100 \%)$ & & \\
Steroid use & $75 / 90(83.33 \%)$ & & \\
AZA use & $15 / 90(16.67 \%)$ & NA & \\
CSA use & $9 / 90(10.00 \%)$ & & \\
MMF use & $16 / 90(17.78 \%)$ & & \\
\hline
\end{tabular}

Data are shown as number or median (interquartile range), respectively. SLE: systemic lupus

erythematosus; SLEDAI: systemic lupus erythematosus disease activity index; LDL:

low-density lipoprotein; HDL: high-density lipoprotein; CIMT: carotid intima-media thickness;

HCQ: Hydroxychloroquine; AZA: Azathioprine; CSA: Cyclosporin A; MMF: Mycophenolate

Mofetil.

Methods: 90 SLE patients and 50 healthy controls were included. According to carotid intima-media thickness (cIMT), SLE patients were further divided into SLE-AS and SLE-NonAS subgroups (cut-off point: $1.0 \mathrm{~mm}$ ). Traditional CVD risk factors, inflammatory biomarkers and PCSK9 concentrations were compared between: (I) SLE patients and controls; (II) SLE-AS subgroup and SLE-NonAS subgroup. Correlational analysis and multivariate linear regression analysis were applied to analyze the association between PCSK9 levels and disease parameter and the predictors of PCSK9 levels in SLE patients. Effects on PCSK9 concentrations by monotherapy with hydroxychloroquine (HCQ), which is thought having protective effects against CVD in SLE, were investigated by follow-up analysis in 15 SLE patients with inactive disease (SLEDAI=2). In animal experiment, murine model of SLE with AS was set up by intraperitoneally injection of lipopolysaccharides (LPS) in ApoE-/- mice. 30 female ApoE-/- mice were respectively administrated with LPS (SLE+AS group, $n=10$ ), saline (AS group, $n=10$ ) and LPS plus injection of lentiviruse-PCSK9 small hairpin RNA targeting the mouse PCSK9 gene into the tail vein to interfere PCSK9 expression (SLE+AS+PCSK9i group, $\mathrm{n}=10$ ). 10 female C57BL/6 mice were included as controls. Serum concentrations of PCSK9 and inflammatory biomarkers including TNF- $\alpha$ and IL-1 $\beta$, atherosclerotic lesion, lipids parameters, expression of PCSK9, TLR4 and NF- $\mathrm{KB}$ p65 in atherosclerotic plaque were assessed.

Results: Characteristics of SLE patients and controls were listed in Table 1. SLE patients had significantly elevated serum PCSK9 levels than controls, especially in SLE-AS subgroup, accompanied with higher ratio of cIMT thickening. Correlationa analysis showed PCSK9 concentrations correlated with C-reactive protein (CRP) levels, age and erythrocyte sedimentation rate (ESR), but not lipids parameters Univariate and multivariate linear regression revealed that only CRP, but not age or ESR was positive predictors of PCSK9. Monotherapy with HCQ for 3 months significantly reduced PCSK9 levels in inactive SLE patients (Table 2, Figure 1). Mice in SLE+AS group had significantly higher serum PCSK9 concentrations than mice in AS group and C57BL/6 mice. Immunohistochemistry showed that PCSK9 overexpression was observed in SLE+AS mice than those in AS group and SLE+AS +PCSK9i group. Mice in SLE+AS+PCSK9i group exhibited decreased inflammatory cell infiltration in atherosclerotic plaque, alleviated atherosclerotic lesion, lower serum TNF- $\alpha$ and IL-1 $\beta$ levels and attenuated expression of TLR4 and NF- $\mathrm{BB}$ p65 in atherosclerotic plaque than SLE+AS group. PCSK9 silencing had no significant effects on lipids parameters in SLE+AS mice (Figure 2).

Table 2 Multivariate linear regression of CRP, ESR, age (independent variable) and PCSK9 (dependent variable) in SLE patients

\begin{tabular}{llcccc}
\hline \multicolumn{5}{c}{ Unstandardized Coefficients } & \multicolumn{4}{l}{ Standardized Coefficients } \\
Model & B & Std. Error & Beta & t & $p$ \\
\hline (Constant) & 280.339 & 72.128 & & 3.887 & $<0.001$ \\
CRP & 4.040 & 0.972 & 0.408 & 4.158 & $<0.001$ \\
ESR & 1.028 & 0.974 & 0.103 & 1.055 & 0.294 \\
Age & 2.931 & 1.767 & 0.159 & 1.659 & 0.101 \\
\hline
\end{tabular}

SLE: systemic lupus erythematosus; PCSK9: Proprotein convertase subtilisin/kexin type 9; CRP: C-reactive protein; ESR: erythrocyte sedimentation rate.
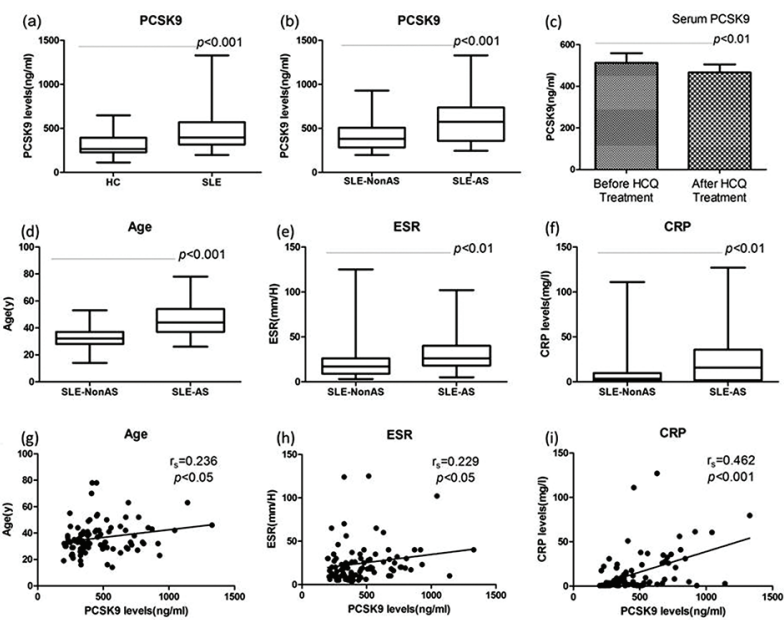

Figure 1. (a)-(b) SLE patients had significantly elevated serum PCSK9 concentrations than controls, especially in those with AS; (c) Monotherapy with HCQ for three months significantly reduced PCSK9 levels in SLE patients with inactive disease $(n=15)$; (d)-(f) Difference of age, ESR and CRP levels between patients in SLE-AS subgroup and those in SLE-NonAS subgroup had statitical significance; (g)-(i) Correlational analysis showed that serum PCSK9 levels correlated with age, CRP levels and ESR in SLE patients. 